\title{
Annexin A7 expression is downregulated in late-stage gastric cancer and is negatively correlated with the differentiation grade and apoptosis rate
}

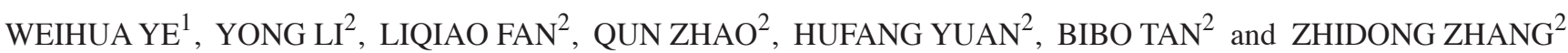 \\ ${ }^{1}$ Department of Ultrasound and ${ }^{2}$ The Third Department of Surgery, \\ The Fourth Hospital of Hebei Medical University, Shijiazhuang, Hebei 050011, P.R. China
}

Received August 3, 2016; Accepted January 16, 2018

DOI: $10.3892 / \mathrm{ol} .2018 .8576$

\begin{abstract}
Annexin A7 is a member of the Annexin A family, which participates in various biological processes. Accumulating evidence has demonstrated that Annexin A7 serves an important role in tumorigenesis and is dysregulated in multiple types of cancer. However, the role of Annexin A7 in the tumorigenesis of gastric cancer remains to be determined. The present study revealed that Annexin A7 expression is downregulated in late-stage gastric cancer and is negatively correlated with the differentiation grade and apoptosis. There was a significant difference in Annexin A7 mRNA and protein expression in gastric cancer samples with distinct differentiation grades, with the lowest expression being observed in the highly differentiated cases. A terminal deoxynucleotidyl-transferase-mediated dUTP nick end labelling assay demonstrated that the apoptosis indices of highly, moderately and poorly differentiated gastric cancers were $18.12 \pm 2.40$, $9.73 \pm 1.73$ and $4.13 \pm 0.83 \%$, respectively, with statistical significance $(\mathrm{P}<0.05)$. Flow cytometric analysis demonstrated that the apoptosis rates of gastric cancer MKN74, SGC7901 and BGC823 cells were $10.07 \pm 1.21,7.11 \pm 1.04$ and $4.25 \pm 1.02 \%$, respectively, with statistical significance $(\mathrm{P}<0.05)$. Spearman's rank correlation analysis revealed that the Annexin A7 mRNA and protein levels were negatively correlated with the differentiation grade of the gastric cancer tissues, while the apoptosis index was positively correlated with the differentiation grade of the gastric cancer tissues. Furthermore, the apoptosis index was negatively correlated with Annexin A7 mRNA and protein expression. Similar associations were observed among Annexin A7 expression, differentiation grades and apoptosis in gastric cancer cell lines. The results of the present study
\end{abstract}

Correspondence to: Dr Yong Li, The Third Department of Surgery, The Fourth Hospital of Hebei Medical University, 12 Jiankang Road, Shijiazhuang, Hebei 050011, P.R. China

E-mail: liyong_hbth@163.com

Key words: Annexin A7, gastric adenocarcinoma, apoptosis, differentiation grade, tumor progression, biomarker demonstrated that Annexin A7 expression is downregulated, while apoptosis is upregulated, with the progression of gastric adenocarcinoma. These observations suggested that Annexin A7 may inhibit apoptosis during tumorigenesis and that it is a potential biomarker for the diagnosis, prognosis and treatment of gastric adenocarcinoma.

\section{Introduction}

Gastric cancer is one of the most common types of malignancy worldwide and was the second leading cause of cancer-associated morbidity and mortality in China in 2015 (1). The main treatment for gastric cancer is surgery; however, due to the absence of symptoms in early gastric cancer, as well as the lack of simple and sensitive screening methods, over half of patients are diagnosed at advanced stages and have missed the opportunity for radical surgery. Therefore, it is important to identify and validate biomarkers for the early diagnosis of gastric cancer.

Annexin is a member of the calcium ion-dependent phospholipid-binding protein superfamily that contains A, B, C, D and E families, which all have similar chemical structures (2). Annexin has N-terminal calcium- and phospholipid-binding domains, and a conserved C-terminal membrane phospholipid-binding domain. The differences among the family members are the various lengths and sequences of the N-terminal structural domains (3). The Annexins expressed in vertebrate cells are in the Annexin A family, which contains 12 members (Annexin A1 to A11, and A13). Annexin A family members participate in various biological processes, including cytoskeletal structure and activity, pinocytosis, exocytosis, conformation and activation of cell membrane receptors, function of cell transmembrane ion channels, cell signaling, as well as cell division, proliferation, differentiation and apoptosis (3-8). The human Annexin A7 gene was first identified in the 1970s by Creutz et al (9) and is expressed in various tissues $(10,11)$. Annexin A7 regulates cell growth, differentiation, proliferation and apoptosis by inhibiting coagulation and phospholipase A2 activity, promoting cell secretion, accelerating chromaffin particle gathering and modulating cell signal transduction $(4,12-14)$. Annexin A7-knockout mice develop spontaneous tumors (15). 
Furthermore, a number of studies have demonstrated that Annexin A7 serves an important role in tumorigenesis and that it is dysregulated in multiple types of tumor tissue (16). However, the roles of Annexin A7 in tumorigenesis remain inconclusive. Annexin A7 is downregulated in malignant glioma (17), glioblastoma multiform (18), melanoma (19) and prostate cancer (20), but it is upregulated in liver cancer (16), gastric cancer (21), nasopharyngeal carcinoma (22), colorectal cancer (23), cervical squamous cell carcinoma (24) and breast cancer (25). These observations indicated that Annexin A7 may either suppress or promote tumorigenesis, depending on the type of tumor. Therefore, it is important to further elucidate the molecular mechanisms of Annexin A7 in the tumorigenesis of different types of cancer.

Previously, we reported that the expression of Annexin A7 is upregulated in gastric cancer tissues, compared with para-carcinoma tissues, and that a high expression of Annexin A7 is a predictive factor for lymphatic metastasis of gastric cancer (26). However, the expression status of Annexin A7 in different stages of gastric cancer remains unknown. In addition, evasion of apoptosis serves a key role in gastric cancer progression, but the association between Annexin A7 expression and gastric cancer cell apoptosis remains unclear.

In order to assess the associations between Annexin A7 expression, and tumor differentiation and apoptosis, the present study analyzed the expression of Annexin A7 and detected apoptosis in gastric cancer clinical tissues and cell lines with various differentiation grades through reverse transcription-quantitative polymerase chain reaction (RT-qPCR), western blot analysis, terminal deoxynucleotidyl transferase-mediated dUTP nick end labeling (TUNEL) and flow cytometry experiments.

\section{Materials and methods}

Cell lines and reagents. The highly differentiated human gastric adenocarcinoma MKN74 cell line, the moderately differentiated human gastric adenocarcinoma SGC7901 cell line and the poorly differentiated human gastric adenocarcinoma BGC823 cell line were provided by the Fourth Hospital of Hebei Medical University (Hebei, China). All cell lines were maintained in RPMI-1640 medium (Invitrogen; Thermo Fisher Scientific, Inc., Waltham, MA, USA) containing $10 \%$ fetal bovine serum (Hangzhou Sijiqing Biological Engineering Materials Co., Ltd., Hangzhou, China), 100 U/ml penicillin and $100 \mathrm{mg} / \mathrm{ml}$ streptomycin in a saturated humidity incubator with $5 \% \mathrm{CO}_{2}$ at $37^{\circ} \mathrm{C}$.

Collection and preparation of gastric cancer specimens of various differentiation grades. A total of 85 fresh gastric cancer specimens were collected from patients who had been diagnosed with gastric cancer and had undergone either radical distal gastrectomy, proximal subtotal gastrectomy or total gastrectomy in the Department of General Surgery of the Fourth Hospital of Hebei Medical University between January 2013 and March 2014. Of the patients, 49 were male and 36 were female, with a mean age of 55 \pm 6.2 years (range: $39-79$ years). Patients who also had other malignancies and had received preoperative radiotherapy, chemotherapy or biotherapy were excluded. The patients were confirmed as having gastric adenocarcinoma through postoperative pathology, and the numbers of highly, moderately and poorly differentiated gastric adenocarcinoma cases were 23, 30 and 32, respectively. Each specimen was divided into three sections following surgical removal, and those used for RT-qPCR and western blot analysis were immediately placed into liquid nitrogen for quick freezing, and were subsequently transferred and stored at $-80^{\circ} \mathrm{C}$. The tissues used for TUNEL staining were fixed with $4 \%$ paraformaldehyde at room temperature for $48 \mathrm{~h}$, embedded in paraffin and routinely sliced (5- $\mu \mathrm{m}$ thick). The aforementioned study protocol was approved by the Ethics Committee of the Fourth Hospital of Hebei Medical University and all patients provided preoperative written informed consent.

$R T-q P C R$. Total RNA was extracted from tissues and cells using the TRIzol one-step method (Invitrogen; Thermo Fisher Scientific, Inc.), and the integrity, content and purity of the RNA was detected through agarose gel electrophoresis and ultraviolet spectroscopy, respectively. Total RNA (5 $\mu \mathrm{g}$ ) was reverse transcribed to cDNA using an M-MLV reverse transcription kit (Promega Corporation, Madison, WI, USA), and qPCR was conducted using SYBR-Green (Promega, Corporation) on a fluorescence quantitative PCR machine (Applied Biosystems; Thermo Fisher Scientific, Inc.). The reaction conditions were as follows: Pre-denaturation at $95^{\circ} \mathrm{C}$ for $5 \mathrm{~min}$; 40 cycles of denaturation at $95^{\circ} \mathrm{C}$ for $15 \mathrm{sec}$, annealing at $60^{\circ} \mathrm{C}$ for $34 \mathrm{sec}$, and extension at $72^{\circ} \mathrm{C}$ for $25 \mathrm{sec}$; followed by denaturation at $95^{\circ} \mathrm{C}$ for $15 \mathrm{sec}$, annealing at $57^{\circ} \mathrm{C}$ for $1 \mathrm{~min}$, and termination with extension at $95^{\circ} \mathrm{C}$ for $30 \mathrm{sec}$. The primer sequences were as follows: Annexin A7 forward, 5'-GTATCCACAGCCACC TTCACAGTC-3' and reverse, 5'-CGCTGAGTACGTCGTGGA GTC-3'; and GAPDH forward, 5'-CGCTGAGTACGTCGT GGAGTC-3' and reverse, 5'-GCTGATGATCTTGAGGCT GTTGTC-3'. The PCR primers were synthesized by Beijing Dingguo Changsheng Biotechnology Co., Ltd. (Beijing, China). GAPDH was used as the internal reference gene, and the expression level of mRNA of the target gene was measured by the quantitative cycle $(\mathrm{Cq})$ method (27), the formulas of which were as follows: $\Delta \mathrm{Cq}$ (test) $=\mathrm{Cq}$ (target, test)-Cq (ref, test); and $\Delta \mathrm{Cq}$ (calibrator) $=\mathrm{Cq}$ (target, calibrator) $-\mathrm{Cq}$ (ref, calibrator). The $\Delta \mathrm{Cq}$ value of the calibrator was used to normalize that of the test, which was $\Delta \Delta \mathrm{Cq}=\Delta \mathrm{Cq}$ (test) $-\Delta \mathrm{Cq}$ (calibrator). Finally, the relative expression (RQ) of the target gene was calculated by $R Q=2^{-\Delta \Delta C q}$. The value of $2^{-\Delta \Delta C q}$ in the measurement group was set as 1 ; therefore, the multiple proportion relations of other experimental groups relative to the measurement group were obtained $(27,28)$. The mean of three experiments was taken as the actual value of each sample.

Western blot analysis. Total protein was extracted from the gastric cancer tissues and cell lines using radioimmunoprecipitation assay lysis buffer (1\% Nonidet P-40, $0.5 \%$ sodium deoxycholate and $0.1 \%$ SDS in $1 \mathrm{x}$ phosphate buffer solution) containing protease inhibitors $(2 \mu \mathrm{g} / \mathrm{ml}$ aprotinin, $2 \mu \mathrm{g} / \mathrm{ml}$ leupeptin and $1 \mathrm{M}$ phenylmethane sulfonyl fluoride) for $30 \mathrm{~min}$ on ice. The protein concentration was determined using a bicinchoninic acid assay. Equal amounts of proteins $(10 \mu \mathrm{g})$ were separated by $10 \%$ SDS-PAGE, prior to being transferred onto nitrocellulose membranes. The membranes were blocked in $2 \%$ nonfat milk in Tris-buffered saline (TBS) for $1 \mathrm{~h}$ at 
room temperature. The membranes were incubated with a rabbit anti-human Annexin A7 primary antibody (dilution, 1:1,000; 10154-2-AP; ProteinTech Group, Inc., Chicago, IL, USA) or a rabbit anti-human GAPDH primary antibody (dilution, 1:3,000; YT5052; ImmunoWay Biotechnology, Plano, TX, USA) overnight at $4^{\circ} \mathrm{C}$, followed by washing with TBS containing $0.1 \%$ Tween-20 and incubation with an IRDye 680LT-conjugated secondary antibody (dilution, 1:1,000; Goat anti-Rabbit, A11369; Invitrogen; Thermo Fisher Scientific, Inc.) at room temperature in the dark for $2 \mathrm{~h}$. An Odyssey double color infrared laser imaging system (Odyssey Sa Software CD Version 2.0; LI-COR Biosciences, Lincoln, NE, USA) was adopted for scanning and calculating the integral absorbance values of the bands, and the ratio of the gray values of the target band and the corresponding GAPDH band was used to determine the target protein expression.

Detection of apoptosis using a TUNEL assay. Cell apoptosis was detected using a TUNEL apoptosis detection kit (Roche Diagnostics, Basel, Switzerland), according to the manufacturer's protocol. In brief, the paraffin-embedded sections of gastric cancer tissues were dewaxed at $60^{\circ} \mathrm{C}$, washed twice with xylene for 5 min each time, rehydrated in a descending alcohol series $(100,95,90,80$ and $70 \%$ for $5 \mathrm{~min}$ of each alcohol concentration) and processed with proteinase $\mathrm{K}$ at $37^{\circ} \mathrm{C}$ for $20 \mathrm{~min}$, followed by processing with $0.1 \%$ Triton $\mathrm{X}-100$ for 8 min and rinsing with phosphate-buffered saline (PBS) twice, for 5 min each time. After incubation with TUNEL reaction mixture at $37^{\circ} \mathrm{C}$ in the dark for $60 \mathrm{~min}$, the sections were washed with PBS 3 times ( $5 \mathrm{~min} /$ wash). The sections were further counterstained with $5 \mu \mathrm{g} / \mathrm{ml}$ propidium iodide (PI) dye at room temperature for $5 \mathrm{~min}$ and mounted with gum. The number of apoptotic cells per 100 cells in each field of view of five random fields was counted under an inverted fluorescence microscope (magnification, x400; Nikon Corporation, Japan). The apoptosis index (AI) was calculated as follows: $\mathrm{AI}=($ the number of apoptotic cells/total cell count) $\times 100 \%$.

Detection of apoptosis through flow cytometry. Cells $\left(1 \times 10^{5}\right)$ were centrifuged at $111.8 \mathrm{x} \mathrm{g}$ for $5 \mathrm{~min}$ at room temperature and were washed twice with precooled PBS. The cell pellets were resuspended in $100 \mu \mathrm{l}$ of binding buffer, followed by the addition of $5 \mu \mathrm{l}$ of Annexin V-fluorescein isothiocyanate (FITC) and incubation in the dark at room temperature for 15 min. Subsequently, the samples were mixed with $10 \mu 1$ PI and were incubated in the dark at room temperature for 5 min. The samples were analyzed using a flow cytometer (FACSCalibur; BD Biosciences, San Diego, CA, USA) with an excitation wavelength of $488 \mathrm{~nm}$ within $1 \mathrm{~h}$. A band-pass filter with a wavelength of $515 \mathrm{~nm}$ was applied to detect FITC fluorescence, while another filter with a wavelength of $560 \mathrm{~nm}$ was used to detect PI. The apoptosis rate $=$ (the number of early apoptotic cells + the number of late apoptotic cells)/total cell count $\mathrm{x} 100 \%$. The Annexin V-FITC/PI apoptosis detection kit was purchased from BD Biosciences.

Statistical analysis. All statistical analyses were processed using SPSS 19.0 software (IBM Corp., Armonk, NY, USA) and the enumeration data are presented as the mean \pm standard deviation. $\mathrm{P}<0.05$ was considered to indicate a statistically significant difference. One-way analysis of variance was applied for comparisons among groups. With regard to pairwise comparison, the least significant difference test was applied for homogeneity of variance, while Dunnett's T3 test was utilized for heterogeneity of variance. Spearman's rank correlation was used for correlation analysis. The level of significance used was $\alpha=0.05$.

\section{Results}

Annexin A7 expression is downregulated in late-stage gastric adenocarcinoma tissues. In order to investigate the expression status of Annexin A7 in differently differentiated gastric adenocarcinomas, the Annexin A7 mRNA levels in 23, 30 and 32 cases of highly, moderately and poorly differentiated gastric adenocarcinomas, respectively, were determined by RT-qPCR, with GAPDH as the internal control. There was a significantly increased Annexin A7 mRNA expression in the moderately differentiated gastric adenocarcinoma samples, compared with that in the highly differentiated gastric adenocarcinoma samples $(\mathrm{P}<0.05)$. Furthermore, in comparison to expression in the moderately differentiated gastric adenocarcinoma samples, there was a significantly increased expression of Annexin A7 mRNA in the poorly differentiated gastric adenocarcinoma samples $(\mathrm{P}<0.05$; Fig. 1A). Similarly, western blot analysis demonstrated that the Annexin A7 protein levels were downregulated in late-stage gastric adenocarcinoma. There was significantly increased Annexin A7 protein expression in moderately differentiated gastric adenocarcinoma, compared with that in the highly differentiated gastric adenocarcinoma samples $(\mathrm{P}<0.05)$. In comparison to expression in the moderately differentiated gastric adenocarcinoma samples, the Annexin A7 protein level in the poorly differentiated gastric adenocarcinoma samples was significantly increased $(\mathrm{P}<0.05$; Fig. 1B).

Expression of Annexin A7 is downregulated in late-stage gastric adenocarcinoma cell lines. To expand on the aforementioned observations in clinical samples, the Annexin A7 mRNA and protein expression was measured in the poorly differentiated human gastric adenocarcinoma BGC823 cell line, the moderately differentiated human gastric adenocarcinoma SGC7901 cell line, and the highly differentiated human gastric adenocarcinoma MKN74 cell line. The mRNA level of Annexin A7 was significantly increased in the SGC7901 cells, compared with that in the MKN74 cells $(\mathrm{P}<0.05)$, and was significantly increased in the BGC823 cells, compared with that in the SGC7901 cells ( $\mathrm{P}<0.05$; Fig. 2A). In line with this, Annexin A7 protein expression was significantly upregulated in the SGC7901 cells, compared with that in the MKN74 cells $(\mathrm{P}<0.05)$, and was significantly increased in the BGC823 cells, compared with that in the SGC7901 cells $(\mathrm{P}<0.05$; Fig. $2 \mathrm{~B})$. Taken together, these results demonstrated that Annexin A7 was significantly downregulated in late-stage gastric adenocarcinoma tissues and cell lines, implying that Annexin A7 expression is gradually downregulated with the progression of gastric adenocarcinoma.

Enhanced apoptosis in late-stage gastric adenocarcinoma tissues. Evasion of apoptosis is a hallmark of cancer cells. In 
A
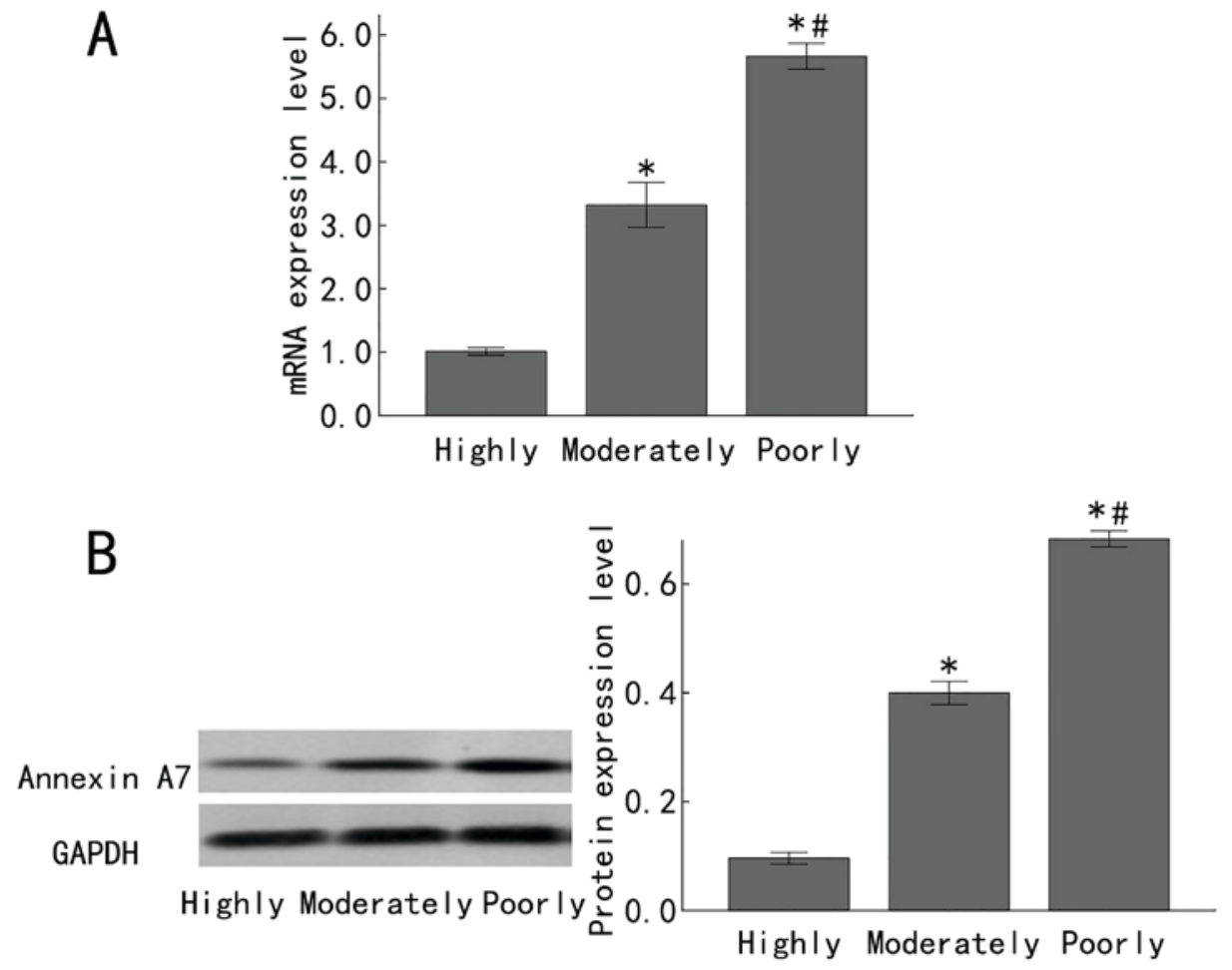

Figure 1. Expression of Annexin A7 in differently differentiated gastric cancer tissues. (A) The expression of Annexin A7 mRNA was measured by fluorescence reverse transcription-quantitative polymerase chain reaction in differently differentiated gastric cancer tissues. (B) The Annexin A7 protein levels in differently differentiated gastric cancer tissues were determined by western blot analysis, with GAPDH as the loading control. Left, representative western blots and right, integrated optical density values of the western blot results. Highly, highly differentiated gastric adenocarcinoma; Moderately, moderately differentiated gastric adenocarcinoma; Poorly, poorly differentiated gastric adenocarcinoma. " $\mathrm{P}<0.05$ compared with the Highly group; ${ }^{\#} \mathrm{P}<0.05$ compared with the Moderately group.

order to assess the potential associations between Annexin A7 expression and apoptosis in gastric cancer and different degrees of differentiation, the apoptosis of gastric cancer tissues at various differentiation grades was detected by a TUNEL assay and fluorescence microscopy (Fig. 3A). The apoptotic cells exhibited green fluorescence, while the PI staining exhibited red fluorescence, representing the total number of cells. A large amount of green fluorescence could be seen in the highly differentiated gastric cancer tissues, indicating a large number of apoptotic cells, while the moderately differentiated gastric cancer tissues exhibited less green fluorescence and poorly differentiated gastric cancer tissues exhibited the least green fluorescence (Fig. 3A). The apoptosis indices in the highly, moderately and poorly differentiated groups were $18.12 \pm 2.40$, $9.73 \pm 1.73$ and $4.13 \pm 0.83 \%$, respectively. The apoptosis index in the poorly differentiated group was significantly decreased compared with that in the moderately differentiated group, and the apoptosis index in the moderately differentiated group was also significantly decreased compared with that in the highly differentiated group (all $\mathrm{P}<0.05$; Fig. 3B). These results demonstrated that the apoptosis index increased with an increase of the differentiation grade of gastric cancer.

Enhancedapoptosis in late-stage gastric adenocarcinoma cell lines. Subsequently, the apoptosis rate in MKN74, SGC7901 and BGC823 cells was analyzed by Annexin V-FITC/PI staining and flow cytometry. The double-variable flow scatter diagram indicates that the number of BGC823 cells in the right lower quadrant (early apoptotic cells) and the right upper quadrant (advanced apoptotic cells) was markedly decreased, while the SGC7901 group exhibited more apoptotic cells and the MKN74 group exhibited the most apoptotic cells (Fig. 4A). The apoptosis rates of the MKN74, SGC7901 and BGC 823 cells were $10.07 \pm 1.21,7.11 \pm 1.04$ and $4.25 \pm 1.02 \%$, respectively. The apoptosis rate in the BGC823 cells was significantly decreased compared with that in the SGC7901 cells $(\mathrm{P}<0.05)$, and the apoptosis rate in the SGC7901 cells was significantly lower than that in the MKN74 cells $(\mathrm{P}<0.05$; Fig. 4B). These results demonstrated that the apoptosis rate was enhanced with the advancement of differentiation of gastric cancer cells.

Association among Annexin A7 expression, differentiation grade and apoptosis rate in gastric cancer tissues and cell lines. Finally, the association among Annexin A7 expression, differentiation grade and apoptosis rate in gastric cancer tissues and cell lines was assessed by Spearman's rank correlation analysis. In gastric cancer clinical samples, the expression of Annexin A7 mRNA and protein was negatively correlated with the differentiation grade of gastric cancer $(\mathrm{r}=-0.926, \mathrm{P}<0.01$ and $\mathrm{r}=-0.950, \mathrm{P}<0.01$, respectively), while the apoptosis index was positively correlated with the differentiation grade $(r=0.949, \mathrm{P}<0.01)$ and the apoptosis index of gastric cancer was negatively correlated with the Annexin A7 mRNA and protein expression ( $\mathrm{r}=-0.978, \mathrm{P}<0.01$ and $\mathrm{r}=-0.973, \mathrm{P}<0.01$, respectively) (Table I; Fig. 5). In the gastric cancer cell lines, the Annexin A7 mRNA and protein expression was negatively correlated with the differentiation 
A
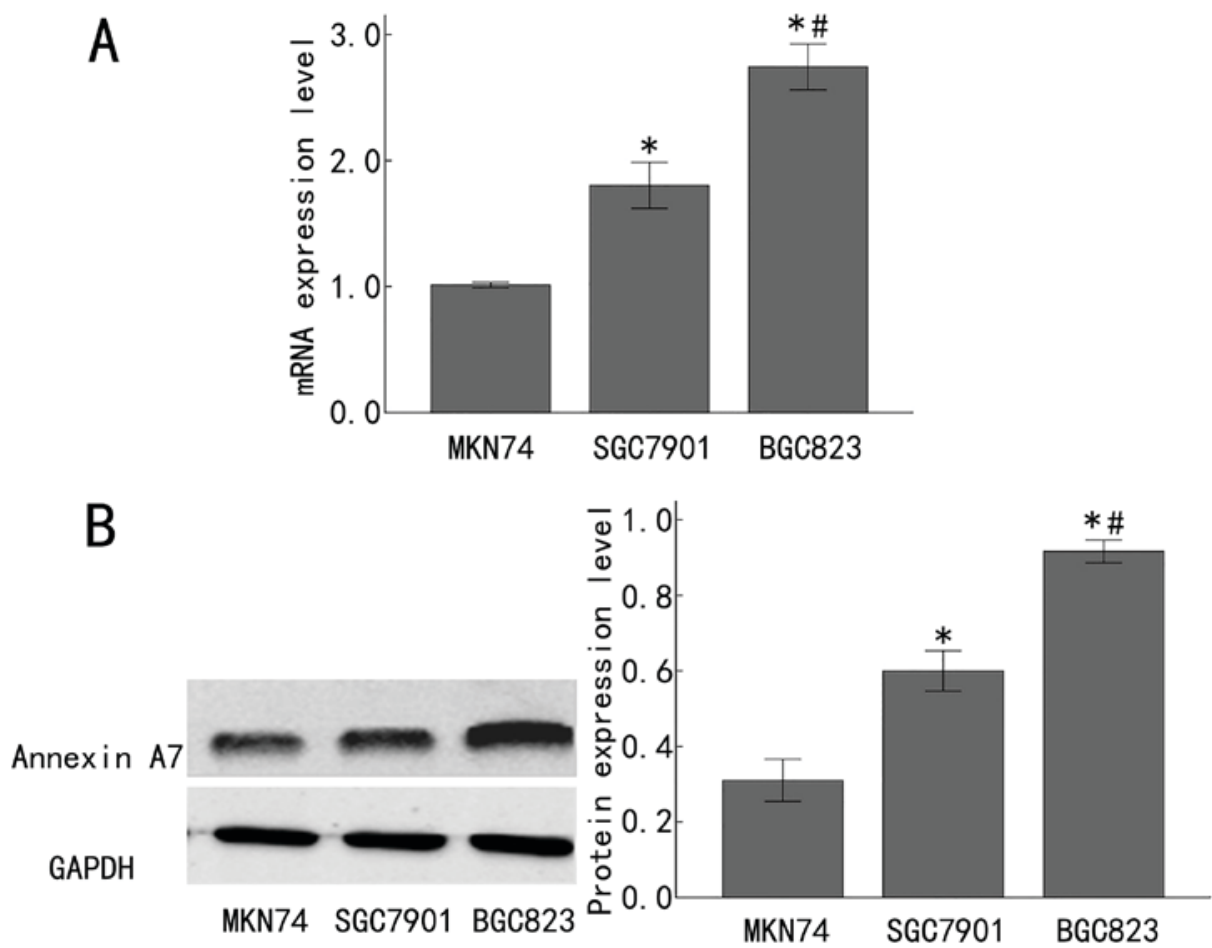

Figure 2. Expression of Annexin A7 in differently differentiated gastric cancer cell lines. (A) The expression of Annexin A7 mRNA was measured by fluorescence reverse transcription-quantitative polymerase chain reaction in differently differentiated gastric cancer cell lines. (B) The Annexin A7 protein levels in differently differentiated gastric cancer cell lines were determined by western blot analysis, with GAPDH as the loading control. Left, representative western blots and right, integrated optical density values of the western blot results. "P $<0.05$ compared with the MKN74 cells; "P<0.05 compared with the SGC7901 cells.

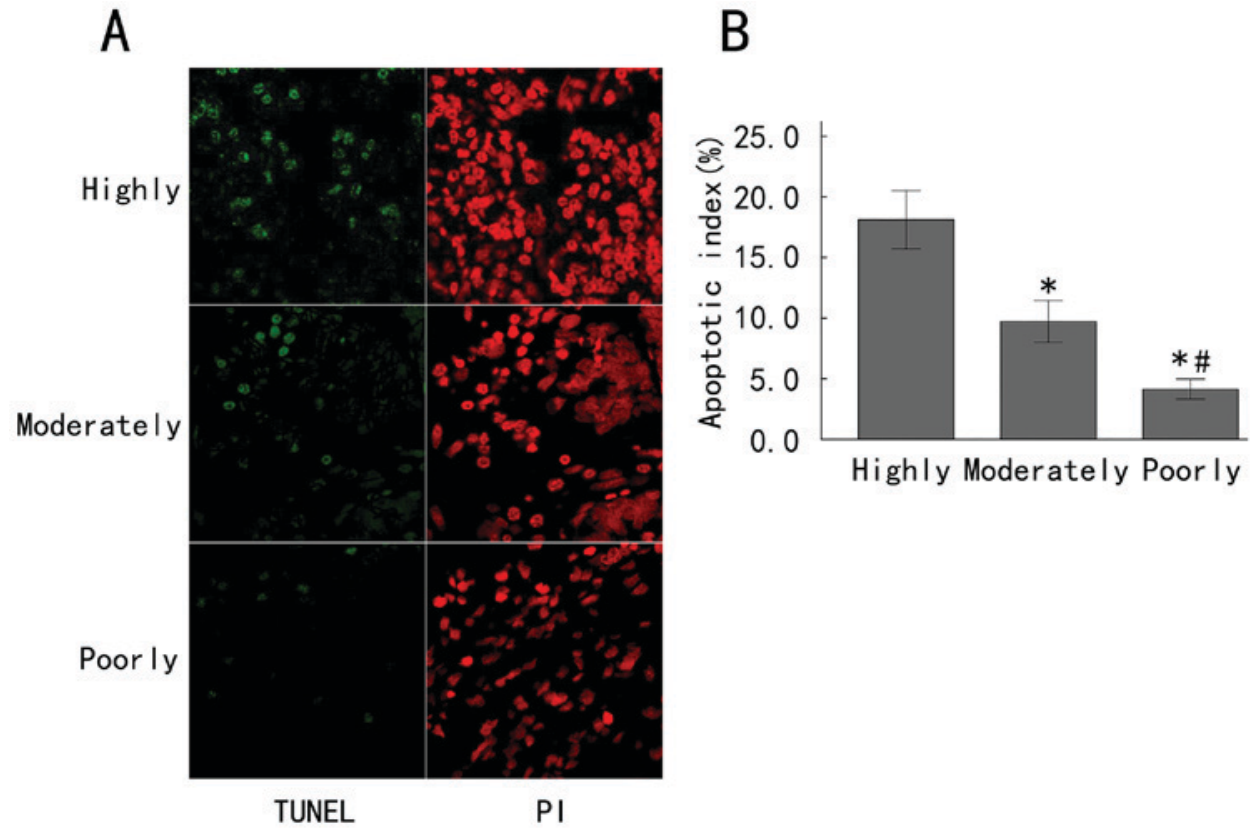

Figure 3. Apoptosis was increased in late-stage gastric adenocarcinoma tissues. (A) The apoptosis of gastric cancer tissues at various differentiation grades was detected by a TUNEL assay and fluorescence microscopy. TUNEL staining exhibited green fluorescence and PI staining exhibited red fluorescence. (B) The number of apoptotic cells was counted per 100 cells in each field of view. The apoptosis index was calculated. Highly, highly differentiated gastric adenocarcinoma; Moderately, moderately differentiated gastric adenocarcinoma; Poorly, poorly differentiated gastric adenocarcinoma. "P<0.05 compared with the Highly group; ${ }^{\prime P}<0.05$ compared with the Moderately group. TUNEL, terminal deoxynucleotidyl transferase-mediated dUTP nick end labeling; PI, propidium iodide.

grade $(\mathrm{r}=-0.934, \mathrm{P}<0.01$ and $\mathrm{r}=-0.938, \mathrm{P}<0.01$, respectively), while the apoptosis rate was positively correlated with the differentiation grade $(\mathrm{r}=0.936, \mathrm{P}<0.01)$. In addition, the apoptosis rate was negatively correlated with the expression of Annexin A7 mRNA and protein ( $\mathrm{r}=-0.917, \mathrm{P}<0.01$ and $\mathrm{r}=-0.933, \mathrm{P}<0.01$, respectively) (Table I; Fig. 6). 
A

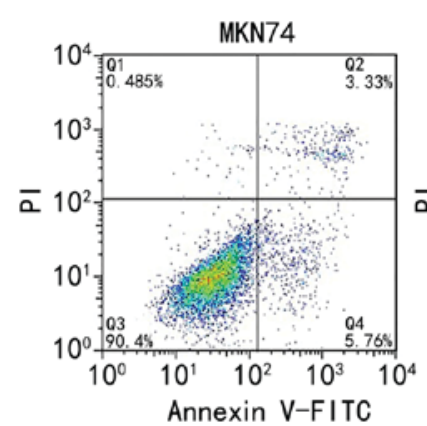

B
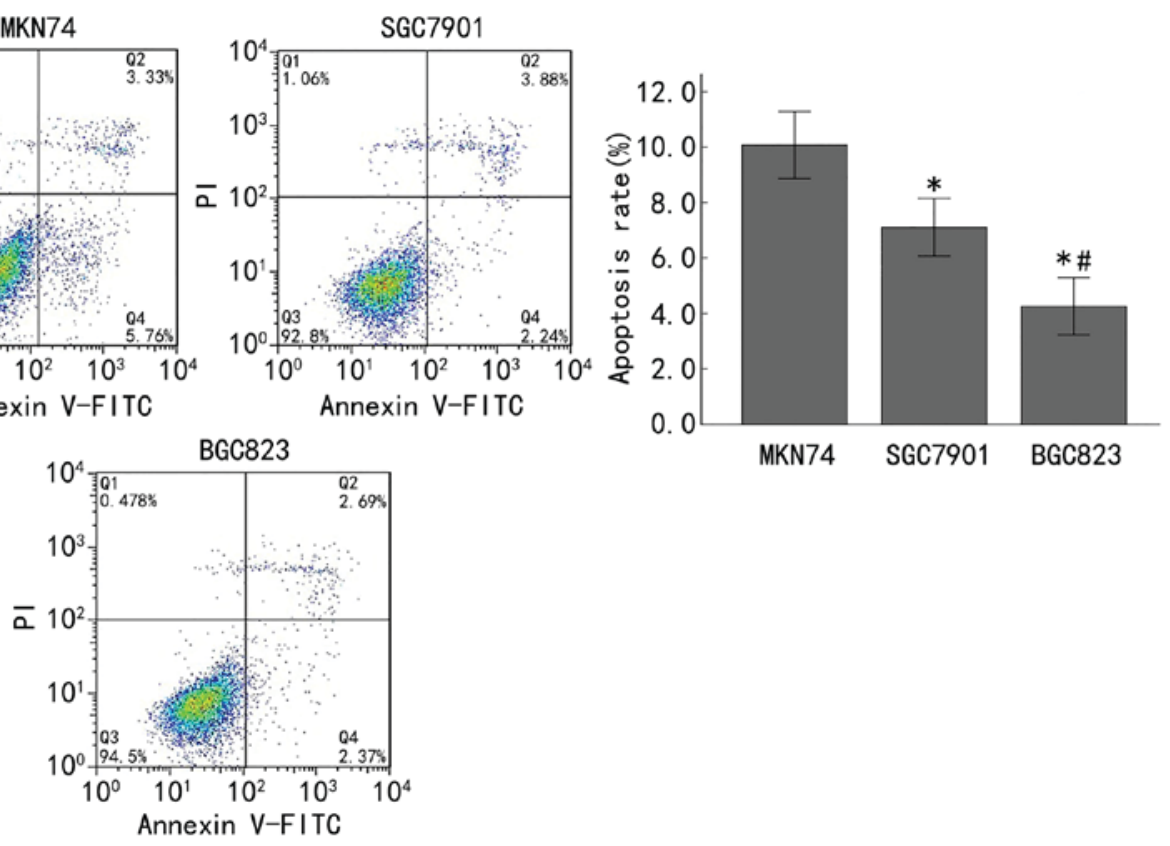

Figure 4. Apoptosis was increased in late-stage gastric adenocarcinoma cell lines. (A) Apoptosis was analyzed using Annexin V-FITC/PI staining and flow cytometry in MKN74, SGC7901 and BGC823 cells. The right lower quadrant demonstrates early apoptotic cells and the right upper quadrant demonstrates late apoptotic cells. (B) Quantification of the percentage of apoptotic cells in MKN74, SGC7901 and BGC823 cells. *P<0.05 compared with the MKN74 cells; ${ }^{\#} \mathrm{P}<0.05$ compared with the SGC7901 cells. FITC, fluorescein isothiocyanate; PI, propidium iodide.

\section{Discussion}

Gastric cancer is an aggressive malignancy with a poor prognosis, and the majority of patients are not suitable for surgery due to the late stage of disease at diagnosis (29). The results of the present study demonstrated that Annexin A7 was significantly downregulated while the rate of apoptosis was increased in late-stage gastric adenocarcinoma tissues and cell lines. Furthermore, Annexin A7 expression was negatively correlated with the differentiation grade and apoptosis rate. These results indicated that Annexin A7 expression is gradually downregulated while apoptosis is gradually upregulated with the progression of gastric adenocarcinoma.

The differentiation grade is a critical factor for tumor prognosis. Highly differentiated tumors have a low malignancy degree and a good prognosis, while poorly differentiated tumors have a high malignancy degree and a poor prognosis. The differentiation degree is also associated with the proliferation, invasion and metastasis of cancer cells; therefore, elucidating the mechanism of tumor differentiation is important in individualizing treatment and prognosis. In the present study, the expression levels of Annexin A7 mRNA and protein in gastric cancer tissues and cell lines with different grades of differentiation were determined. The expression levels of Annexin A7 mRNA and protein were decreased and were negatively correlated with the differentiation grade of gastric cancer, suggesting that Annexin A7 expression increases with the decrease in differentiation grade, further supporting the important role for Annexin A7 in the development of gastric cancer. The results of the present study were consistent with the previous observation that the expression of Annexin A7 may be used as an important predictive indicator for the survival rate of patients with gastric cancer (26). By contrast, Hsu et al (21) reported that the expression of Annexin A7 is associated with the differentiation grade and that positive expression rates exhibited a gradual decrease in highly differentiated tubular and papillary adenocarcinoma, moderately differentiated tubular adenocarcinoma, and poorly differentiated signet ring cell carcinoma and mucinous adenocarcinoma. In addition, $\mathrm{Xi}$ and Zhao (30) have demonstrated that the lower the gastric cancer differentiation grade, the lower the expression of Annexin A7. However, Gong et al (31) reported that differences in the expression of Annexin A7 in highly, moderately and poorly differentiated gastric tubular adenocarcinoma are not statistically significant. This discrepancy may be due to the different methods used to detect Annexin A7 expression and the different sample sizes. A multi-center study with a large sample size and unified methods is required in order to confirm the expression of Annexin A7 in distinctly differentiated gastric cancer.

Apoptosis serves an important role in the physiological and pathological processes of the body, particularly in the development and progression of cancer. Apoptosis is a programmed cell death that involves extracellular and intracellular signals (32). A TUNEL assay, currently the most specific, fastest and most sensitive technique to detect single-cell apoptosis through in situ staining, revealed that highly differentiated gastric cancer exhibited significantly more apoptotic cells, while poorly differentiated gastric cancer exhibited significantly fewer apoptotic cells in clinical gastric tissues. Furthermore, the use of Annexin V-FITC/PI staining with flow cytometry in order to detect gastric cancer cell lines at various differentiation grades revealed that the apoptosis 
Table I. Correlation among Annexin A7 expression, differentiation grade and apoptosis rate in gastric cancer tissues and cell lines was assessed by Spearman's rank correlation analysis.

Variable

r-value

P-value

Gastric cancer tissues

Annexin A7 mRNA expression vs. differentiation grade

Annexin A7 protein expression vs. differentiation grade

$-0.950$

$<0.01$

Apoptosis index vs. differentiation grade

0.949

$<0.01$

Apoptosis index vs. Annexin A7 mRNA expression

$-0.978$

$<0.01$

Apoptosis index vs. Annexin A7 protein expression

$-0.973$

$<0.01$

Gastric cancer cell lines

Annexin A7 mRNA expression vs. differentiation grade

Annexin A7 protein expression vs. differentiation grade
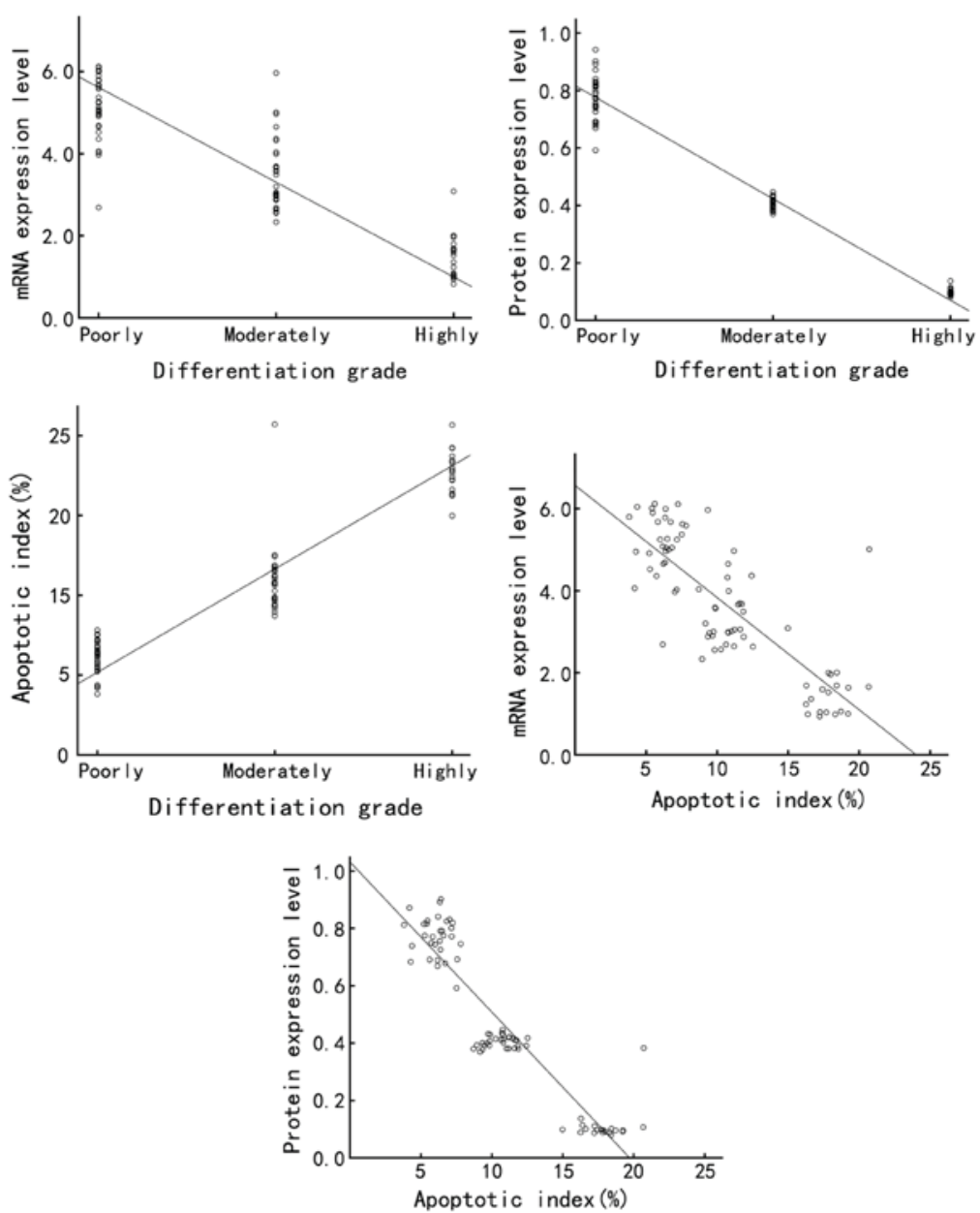

Figure 5. Correlation among Annexin A7 protein expression, differentiation grade and apoptosis index in gastric cancer tissues was assessed by Spearman's rank correlation analysis.

rate of poorly differentiated BGC823 cells was markedly decreased, compared with the rates in highly differentiated MKN74 cells and moderately differentiated SGC7901 cells. These observations in cell lines were consistent with those of TUNEL staining in clinical samples, demonstrating a gradual increase of apoptosis with the advancement of gastric cancer differentiation.

In order to further understand the mechanism of gastric cancer progression and to identify a novel target for treating gastric cancer, the associations among the expression of 

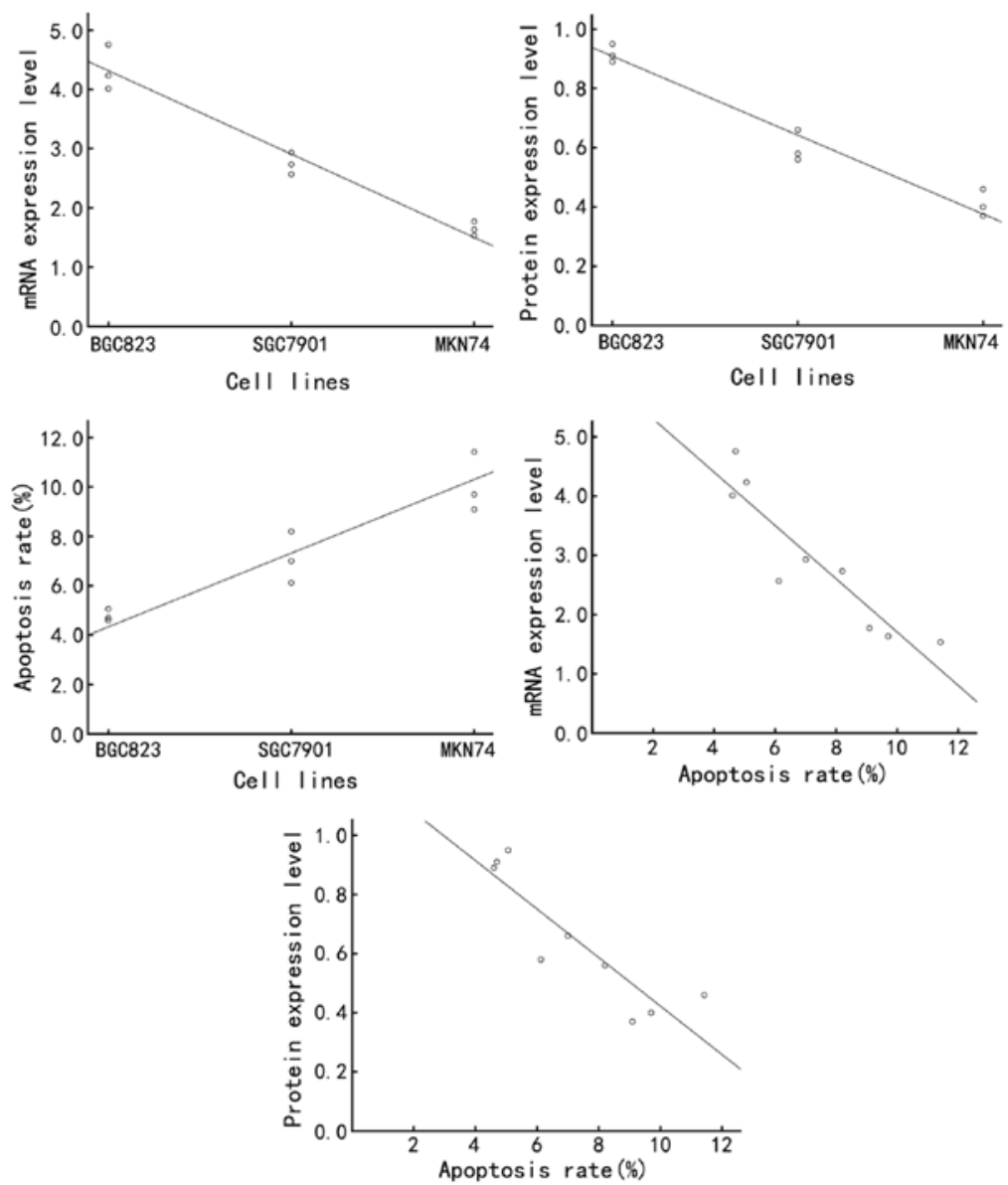

Figure 6. Correlation among Annexin A7 protein expression, differentiation grade and apoptosis rate in gastric cancer cell lines was assessed by Spearman's rank correlation analysis.

Annexin A7, the differentiation grade and the apoptosis rate of gastric cancer cells in gastric cancer tissues and cell lines at various differentiation grades were analyzed. It was revealed that the expression of Annexin A7 was negatively correlated with the differentiation of gastric cancer, while the apoptosis rate was positively correlated with the differentiation of gastric cancer. In addition, Annexin A7 expression was negatively correlated with apoptosis. These results indicated that the expression of Annexin A7 was high and the apoptosis rate was low in poorly differentiated gastric cancer, suggesting that Annexin A7 may inhibit apoptosis during the development and progression of gastric cancer. However, this mechanism requires confirmation by genetic modulation of Annexin A7 expression in vitro and in animal models.

In summary, by analyzing the Annexin A7 expression and the apoptosis rate in gastric cancer tissues and cell lines at various differentiation grades, it was revealed that Annexin A7 expression was gradually downregulated while apoptosis was gradually upregulated with the progression of gastric adenocarcinoma. The results of the present study suggested that Annexin A7 is a potential biomarker for the diagnosis, prognosis and treatment of gastric adenocarcinoma.

\section{Acknowledgements}

Not applicable.

\section{Funding}

No funding was received.

\section{Availability of data and materials}

The datasets used and/or analyzed during the current study are available from the corresponding author on reasonable request.

\section{Authors' contributions}

YL designed the research. WY, LF and QZ collected clinical data and performed the clinical studies. WY, HY and BT performed the experiments. WY, HY and ZZ analyzed the data. YL, WY and BT wrote the manuscript.

\section{Ethics approval and consent to participate}

This research was performed in accordance with the Declaration of Helsinki and approved by the Ethics Committee of The Fourth Hospital of Hebei Medical University.

\section{Consent for publication}

Patients, parents or guardians provided written informed consent for the publication of the present study. 


\section{Competing interests}

The authors declare that they have no competing interests.

\section{References}

1. Chen W, Zheng R, Baade PD, Zhang S, Zeng H, Bray F, Jemal A, Yu XQ and He J: Cancer statistics in China, 2015. CA Cancer J Clin 66: 115-132, 2016.

2. Monastyrskaya K, Babiychuk EB and Draeger A: The Annexins: Spatial and temporal coordination of signaling events during cellular stress. Cell Mol Life Sci 66: 2623-2642, 2009.

3. Gerke V and Moss SE: Annexins: From structure to function. Physiol Rev 82: 331-371, 2002.

4. Gerke V, Creutz CE and Moss SE: Annexins: Linking Ca2+ signalling to membrane dynamics. Nat Rev Mol Cell Biol 6 : 449-461, 2005.

5. Wang KL, Wu TT, Resetkova E, Wang H, Correa AM, Hofstetter WL, Swisher SG, Ajani JA, Rashid A, Hamilton SR and Albarracin CT: Expression of Annexin A1 in esophageal and esophagogastric junction adenocarcinomas: Association with poor outcome. Clin Cancer Res 12: 4598-4604, 2006.

6. Yan GR, Ding W, Xu SH, Xu Z, Xiao CL, Yin XF and He QY: Characterization of phosphoproteins in gastric cancer secretome OMICS 15: 83-90, 2011.

7. Lauritzen SP, Boye TL and Nylandsted J: Annexins are instrumental for efficient plasma membrane repair in cancer cells. Semin Cell Dev Biol 45: 32-38, 2015.

8. Han Y, Ye J, Dong Y, Xu Z and Du Q: Expression and significance of Annexin A2 in patients with gastric adenocarcinoma and the association with E-cadherin. Exp Ther Med 10: 549-554, 2015.

9. Creutz CE, Pazoles CJ and Pollard HB: Identification and purification of an adrenal medullary protein (synexin) that causes calcium-dependent aggregation of isolated chromaffin granules. J Biol Chem 253: 2858-2866, 1978.

10. Selbert S, Fischer P, Pongratz D, Stewart M and Noegel AA: Expression and localization of Annexin VII (synexin) in muscle cells. J Cell Sci 108: 85-95, 1995.

11. Clemen CS, Hofmann A, Zamparelli C and Noegel AA: Expression and localisation of Annexin VII (synexin) isoforms in differentiating myoblasts. J Muscle Res Cell Motil 20: 669-679, 1999.

12. Fatimathas L and Moss SE: Annexins as disease modifiers. Histol Histopathol 25: 527-532, 2010.

13. Huang Y, Du Y,Zhang X, Bai L, Mibrahim M,Zhang J, Wei Y,LiC Fan S, Wang H, et al: Down-regulated expression of Annexin A7 induces apoptosis in mouse hepatocarcinoma cell line by the intrinsic mitochondrial pathway. Biomed Pharmacother 70 : 146-150, 2015.

14. Song MY, Tang JW, Sun MZ, Liu SQ and Wang B: Localization and expression of CLIC1 in hepatocarcinoma ascites cell lines with high or low potentials of lymphatic spread. Zhonghua Bing Li Xue Za Zhi 39: 463-466, 2010 (In Chinese).

15. Srivastava M, Montagna C, Leighton X, Glasman M, Naga S, Eidelman O, Ried T and Pollard HB: Haploinsufficiency of Anx7 tumor suppressor gene and consequent genomic instability promotes tumorigenesis in the Anx7(+/-) mouse. Proc Natl Acad Sci USA 100: 14287-14292, 2003.

16. Srivastava M, Torosyan Y, Raffeld M,Eidelman O, Pollard HB and Bubendorf L: ANXA7 expression represents hormone-relevant tumor suppression in different cancers. Int J Cancer 121: 2628-2636, 2007.
17. Yadav AK, Renfrow JJ, Scholtens DM, Xie H, Duran GE, Bredel C, Vogel H, Chandler JP, Chakravarti A, Robe PA, et al: Monosomy of chromosome 10 associated with dysregulation of epidermal growth factor signaling in glioblastomas. JAMA 302: 276-289, 2009

18. Hung KS and Howng SL: Prognostic significance of Annexin VII expression in glioblastomas multiforme in humans. J Neurosurg 99: 886-892, 2003.

19. Kataoka TR, Ito A, Asada H, Watabe K, Nishiyama K, Nakamoto K, Itami S, Yoshikawa K, Ito M, Nojima H and Kitamura Y: Annexin VII as a novel marker for invasive phenotype of malignant melanoma. Jpn J Cancer Res 91: 75-83, 2000.

20. Torosyan Y, Dobi A, Glasman M, Mezhevaya K, Naga S, Huang W, Paweletz C, Leighton X, Pollard HB and Srivastava M: Role of multi-hnRNP nuclear complex in regulation of tumor suppressor ANXA7 in prostate cancer cells. Oncogene 29: 2457-2466, 2010.

21. Hsu PI, Huang MS, Chen HC, Hsu PN, Lai TC, Wang JL, Lo GH, Lai KH, Tseng CJ and Hsiao M: The significance of ANXA7 expression and its correlation with poor cellular differentiation and enhanced metastatic potential of gastric cancer. J Surg Oncol 97: 609-614, 2008.

22. Yang M and Liang Q: Study the relationship between the expression of Annexin A7 and CT of nasopharyngeal carcinoma. J Chin Clin Med Imaging 22: 6-9, 2011.

23. Alfonso P, Canamero M, Fernández-Carbonié F, Núñez $\mathrm{A}$ and Casal JI: Proteome analysis of membrane fractions in colorectal carcinomas by using 2D-DIGE saturation labeling. J Proteome Res 7: 4247-4255, 2008

24. Chen X, Gao FL, Chang YZ and Li X: Expression of Annexin A7 in human uterine cervical squamous carcinomas and normal tissues. Acta Anatomica Sinica 41: 603-605, 2010.

25. Leighton X, Srikantan V, Pollard HB, Sukumar S and Srivastava M: Significant allelic loss of ANX7region (10q21) in hormone receptor negative breast carcinomas. Cancer Lett 210: 239-244, 2004.

26. Yuan HF, Li Y, Zhao Q, Fan LQ, Tan BB and Ye WH: Expression of Annexin A7 and its clinical significance in differentiation and metastasis of gastric carcinoma. Int J Clin Exp Pathol 7: 6567-6574, 2014.

27. Livak KJ and Schmittgen TD: Analysis of relative gene expression data using real-time quantitative PCR and the 2(-Delta Delta C(T)) method. Methods 25: 402-408, 2001.

28. Karube A, Shidara Y, Hayasaka K, Maki M and Tanaka T: Suppression of calphobindin I (CPB I) production in carcinoma of uterine cervix and endometrium. Gynecol Oncol 58: 295-300, 1995.

29. Kanat O, O'Neil B and Shahda S: Targeted therapy for advanced gastric cancer: A review of current status and future prospects. World J Gastrointest Oncol 7: 401-410, 2015.

30. Xi JM and Zhao Q: Expression of Annexin A7 in gastric cancer tissues and their effects on the differentiation and metastasis of gastric cancer. J Exp Clin Med 9: 726-727, 2010.

31. Gong X, Tang $J$ and Geng X: Expression and significance of Annexin 7 in gastric cancer and lymphatic metastasis. Inter J Pathol Clin Med 29: 369-373, 2009.

32. Giansanti V, Torriglia A and Scovassi AI: Conversation between apoptosis and autophagy: 'Is it your turn or mine?' Apoptosis 16: 321-333, 2011.

This work is licensed under a Creative Commons Attribution-NonCommercial-NoDerivatives 4.0 International (CC BY-NC-ND 4.0) License. 